\title{
DER UMBAU DER UNIVERSITÄTSBIBLIOTHEK GRAZ - EIN BERICHT IN DREI FORTSETZUNGEN. TEIL 3: DIE UNVOLLENDETE VOLLENDUNG*
}

von Werner Schlacher

Zusammenfassung: Dieser Teil des Berichts vom Umbau der Universitätsbibliothek Graz beinhaltet die Phase von der Gleichenfeier im Juli 2018 zur Eröffnung des Gebäudes im September 2019 und die nachfolgende Entwicklung.

Schlagwörter: Universitätsbibliothek Graz; Umbau

\section{THE RECONSTRUCTION OF THE UNIVERSITY LIBRARY OF GRAZ - A REPORT IN THREE SEQUELS. PART 3: THE INCOMPLETE COMPLETION}

Abstract: This part of the report refers to the period from the topping-out ceremony in July 2018 until the opening of the building in September 2019 and the following development.

Keywords: University Library of Graz; reconstruction

DOI: https://doi.org/10.31263/voebm.v73i1.3471

(c) Werner Schlacher

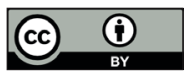

Dieses Werk ist - exkl. einzelner Logos und Abbildungen - lizenziert unter einer Creative-Commons-Lizenz Namensnennung 4.0 International 


\section{Der Wettlauf mit der Zeit}

Obwohl bereits im Winter 2017/18 offensichtlich war, dass die vorausberechneten Termine kaum einzuhalten sein werden, wurde seitens der Bauleitung sehr lange daran festgehalten. Das bedeutete, dass die bereits einmal um ein halbes Jahr verschobene Gleichenfeier nunmehr im Frühsommer 2018 stattfinden sollte, und der Meilenstein „Dach und Fassade dicht" mit Jahresende 2018 erreicht würde. Für die erste Jahreshälfte 2019 waren der Innenausbau und die Möblierung vorgesehen und um den Beginn der Sommerferien sollte dann die Eröffnung des Gebäudes vorgenommen werden. Die Rückübersiedlung der Bibliothek samt den dort beschäftigten Personen und den Beständen sollte kurioserweise in den darauffolgenden Monaten Juli bis September durchgeführt werden, also erst nach der offiziellen Inbetriebnahme.

Da eine abermalige Verschiebung der Gleichenfeier wegen des damit verbundenen Imageverlusts nicht infrage kam, entstand die bizarre Situation, dass am 29. Juni 2018 etwas gefeiert wurde, was augenscheinlich bei Weitem noch nicht erreicht worden war.

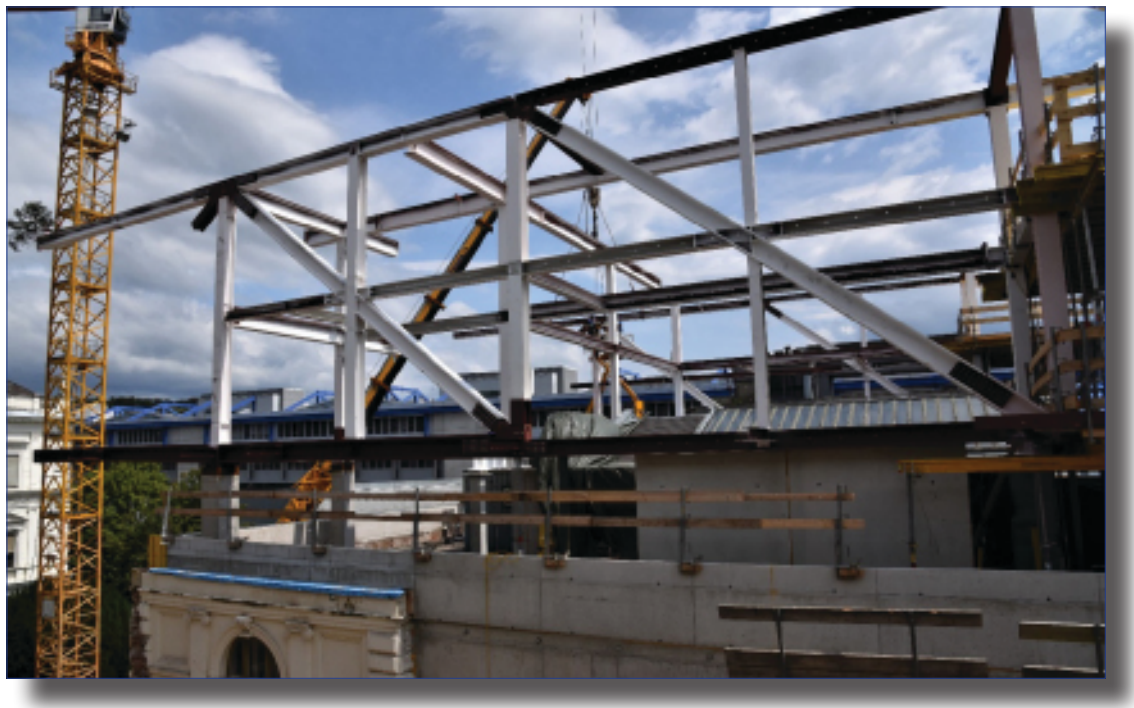

Abb. 1: Blick vom Hauptgebäude der Universität auf die Stahlkonstruktion des Glasriegels am Tag der Gleichenfeier (Foto: Universitätsbibliothek, Robert Ramschak)

Wie an der Abbildung leicht zu erkennen ist, fehlen dem Gebäude zu diesem Zeitpunkt weitgehend jene Merkmale, die für gewöhnlich bei der Abhaltung 
einer so traditionellen Feierlichkeit zu erwarten sind. Die massive Stahlkonstruktion der 18,75 Meter langen und 25 Meter breiten Auskragung war nur etwa zur Hälfte vorhanden, und es erforderte in Wirklichkeit noch etwa drei Monate, bis die die Montage des Stahlskeletts abgeschlossen war. In dieser Zeit kursierten auch immer wieder Gerüchte, dass die Horizontalträger nicht exakt waagrecht ausgerichtet seien. Es wurden mehrere Messungen vorgenommen und einmal sogar kurzzeitig die im Norden vorbeiführende Straße gesperrt. Schließlich einigte man sich auf die Sprachregelung, dass die Abweichung innerhalb der zulässigen Toleranzbreite liege.

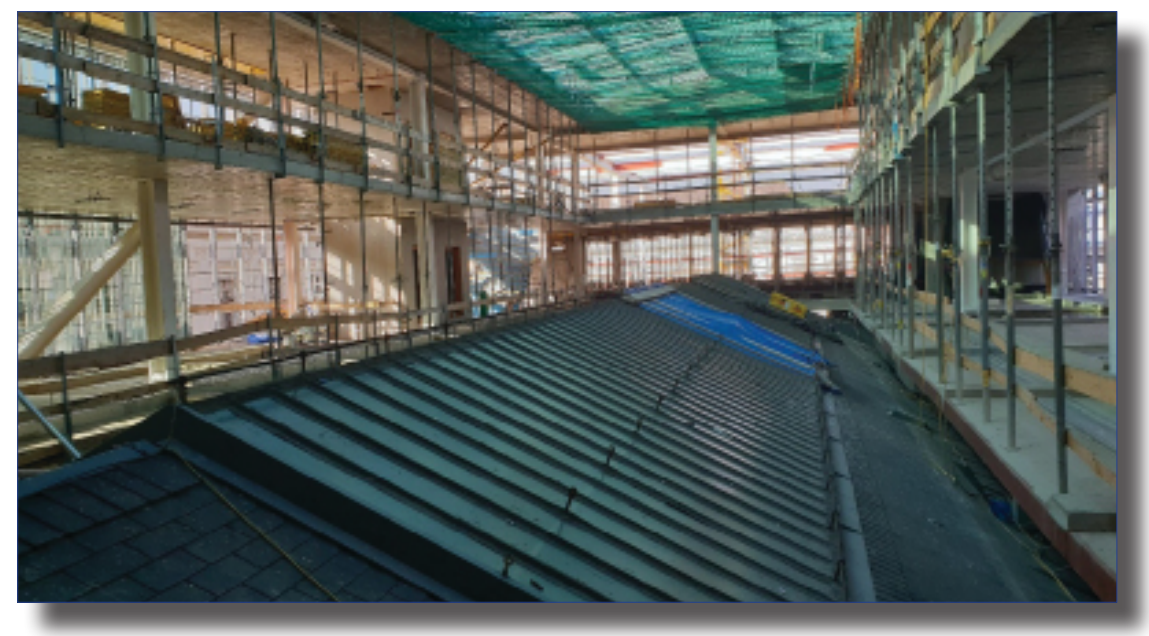

Abb. 2: Ansicht vom Inneren des Glasriegels vom 28.11.2018 (Foto: Universität Graz, Konstantin Tzivanopoulos)

Als nächster für die Fertigstellung des Gebäudes maßgeblicher Termin wurde der Jahreswechsel 2018/19 angegeben. Mit Anfang Jänner 2019 sollten Dach und Fassade geschlossen sein, damit die Ausbauarbeiten im Inneren unbeeinflusst von Witterungsverhältnissen vonstattengehen könnten. Da auch dieses Etappenziel deutlich verfehlt wurde, entschloss man sich nach langem Zögern, den geplanten Eröffnungstermin doch von Juni auf August oder September 2019 zu verlegen. Der Bibliothek wurde versichert, dass die neuen Magazine mit Ende Juni bezugsfertig sein würden, damit nach einer zweimonatigen Phase mit Klimamessungen die Rückübersiedlung zumindest der rezenten Bestände während der Sommermonate durchgeführt werden könne.

Auch diese Prognose erschien von Anfang an ziemlich gewagt, denn eine deutliche Intensivierung der Bautätigkeit war erst ab Mai 2019 zu erkennen. Ab diesem Zeitpunkt waren ständig eine ganze Reihe von Gewerken mit 
Dutzenden Arbeiterlnnen vor Ort und bei diversen Begehungen konnte man erkennen, dass nunmehr tatsächlich die Innenraumgestaltung in Angriff genommen worden war. Trotz der vermehrten Anstrengungen war aber recht bald abzusehen, dass eine Rückübersiedlung der Magazinsbestände in den Sommermonaten nicht zu bewerkstelligen sein würde, da wegen der Präsenz der zahlreichen FirmenmitarbeiterInnen und der regen Bautätigkeit in allen Räumlichkeiten keine seriösen Klimamessungen vorgenommen werden konnten. Außerdem waren bis kurz vor dem neu festgesetzten Eröffnungstermin Ende September die Magazine weder gereinigt noch verschließbar, und auch die technischen Anlagen zur Klimatisierung wurden erst unmittelbar davor eingeschaltet. Ebenso konnte die für einen geregelten Bibliotheksbetrieb unverzichtbare Buchförderanlage nicht zeitgerecht fertiggestellt werden und stand auch noch einige Zeit nach der Eröffnung nicht zur Verfügung.

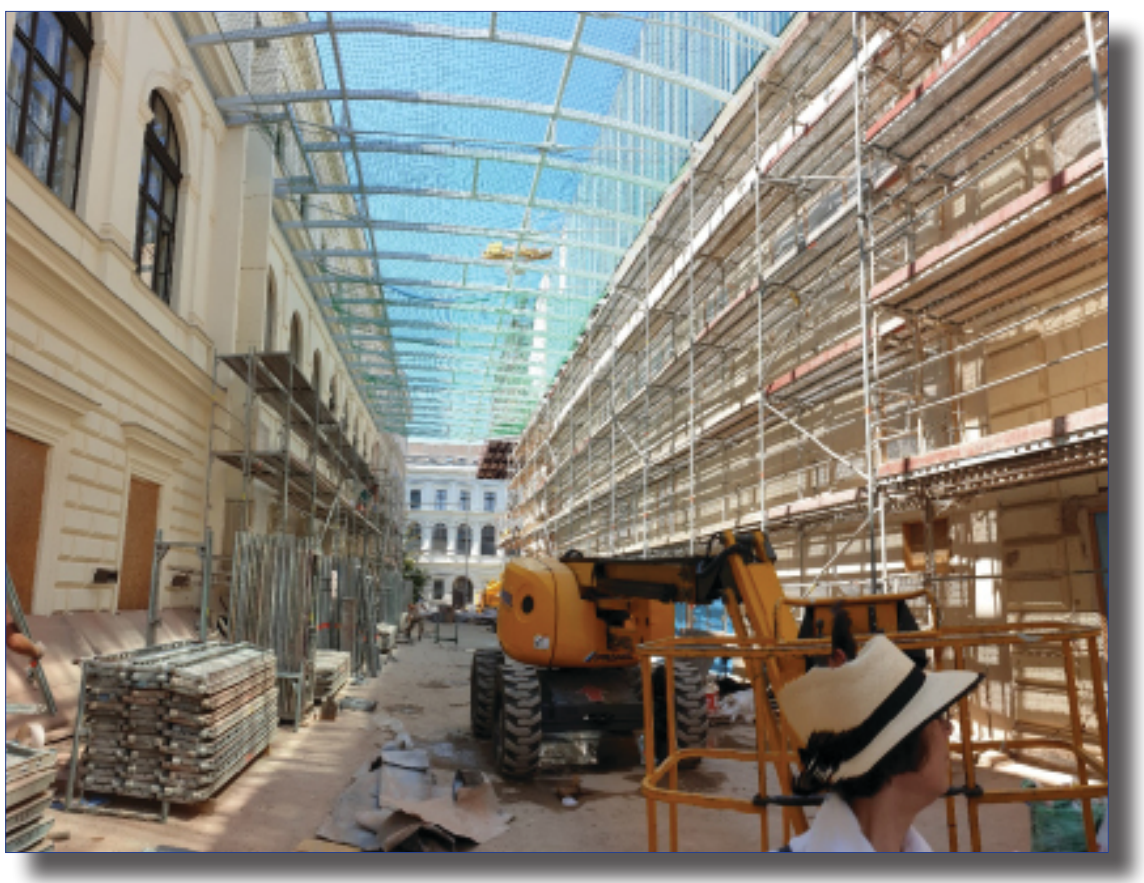

Abb. 3: Ansicht von der Errichtung des neuen Foyers zwischen Uni-Hauptgebäude links und historischem Bibliothekstrakt rechts vom 28. Juni 2019 (Foto: Universitätsbibliothek, Robert Ramschak)

Insgesamt kann festgehalten werden, dass speziell in den Sommermonaten 2019 ein koordiniertes Vorgehen auf der Baustelle nicht mehr zu erkennen 
war. Die chaotischen Umstände reichten von Zufahrten, die durch andere Zulieferer blockiert waren, über Malfarbe, die aus Kübeln auf frisch verlegte Teppiche schwappte, bis zu montierten Deckenelementen, die wieder abgenommen werden mussten, weil die Elektriker mit der Verlegung der darüberliegenden Leitungen noch nicht fertig waren, um nur einige Beobachtungen hier anzuführen. Je näher der 26. September rückte, also jener Tag, der wegen des mit Beginn des Wintersemesters zu vollziehenden Rektoratswechsels für die Eröffnung ausersehen worden war, desto größer wurde die Anspannung aller Beteiligten und der Druck auf die Firmen, bis zu diesem Zeitpunkt ein einigermaßen präsentables Gebäude herzustellen.

\section{Der "große“ Tag}

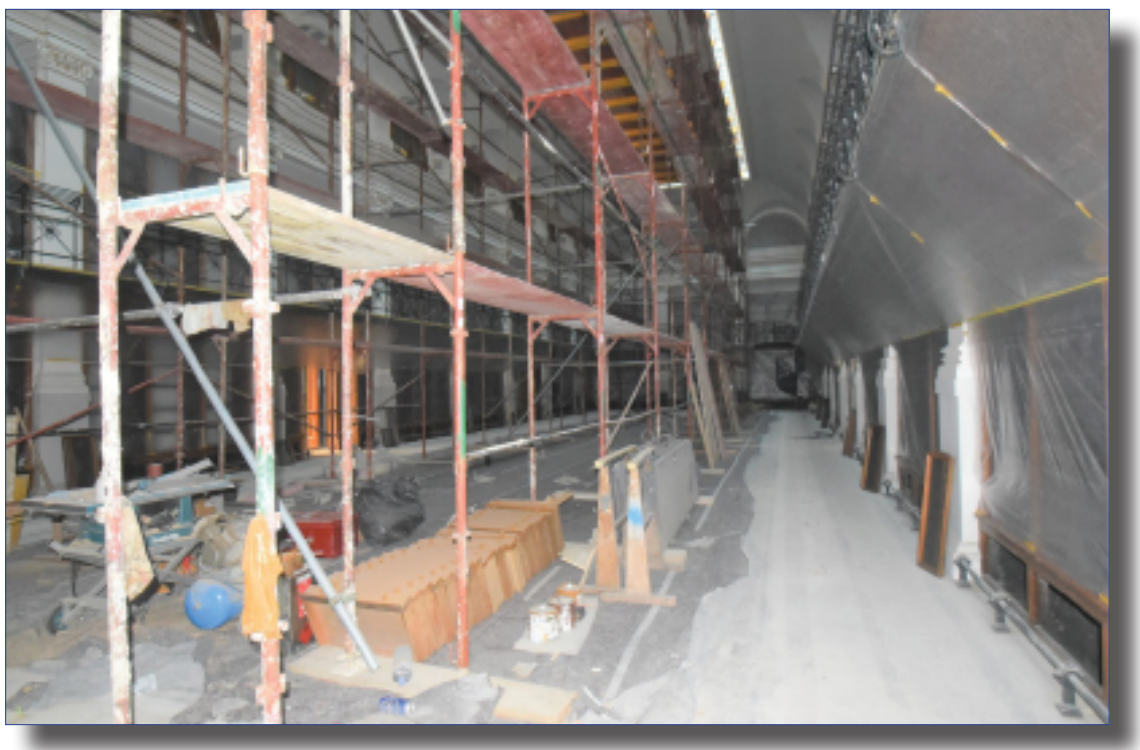

Abb. 4: Der historische Lesesaal drei Wochen vor der Eröffnung (Foto: Universitätsbibliothek, Robert Ramschak)

Auch die Bibliothek wurde in diese Inszenierung miteinbezogen. Da man beim Rundgang mit den geladenen Honoratioren und PressevertreterInnen nicht vor völlig leeren Regalen stehen wollte, mussten Teilbereiche der Freihandbestände aus den Ersatzquartieren in halbfertige Räume übersiedelt werden. Die Bestückung des historischen Lesesaales mit rund 100.000 Bänden musste jedoch wegen des nicht fertiggestellten Glasdaches kurz- 
fristig abgesagt werden. Für die Eröffnung wurde der Lesesaal provisorisch mit den völlig restaurierten Originalmöbeln von 1895 ausgestattet. Erhebliche Mehrkosten, die durch den notwendigen Ab-und neuerlichen Aufbau des Gerüstes und die mehrfache Reinigung des Saales entstanden, wurden dabei in Kauf genommen.

Für die publikumswirksame Eröffnungsveranstaltung am 26. September 2019 wurde mit großem Einsatz versucht, das gesamte Areal in einen dem Festakt angemessenen Zustand zu versetzen. Aus diesem Grund wurde ohne Wissen der Bibliotheksleitung in der Nacht davor eine Reinigung des umliegenden Freigeländes mit einem Spritzwagen veranlasst, was zur Folge hatte, dass am Morgen der Eröffnung ein massiver Wassereintritt im Tiefspeicher der Bibliothek und in mehreren Nebenräumen gravierenden Schaden anrichtete.

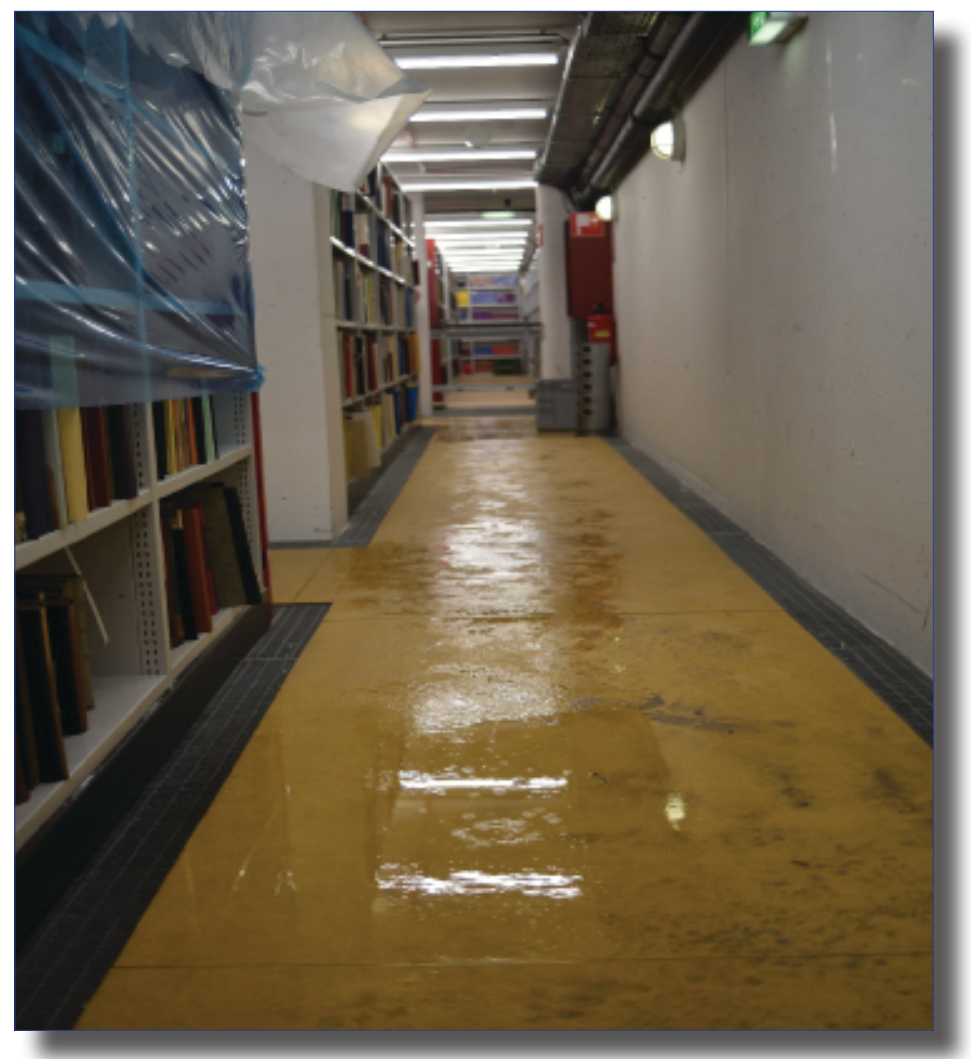

Abb. 5: Wassereintritt am Tag der Eröffnung (Foto: Universitätsbibliothek, Robert Ramschak) 
Parallel zu den Notbergungen, Sicherungs- und Aufräumarbeiten im Kellergeschoß fand in den oberen Etagen die von einer Eventagentur im Auftrag der Universitätsleitung gestaltete Eröffnung der neuen Bibliothek inklusive des in das Gebäude integrierten Hörsaales mit 430 Sitzplätzen statt.

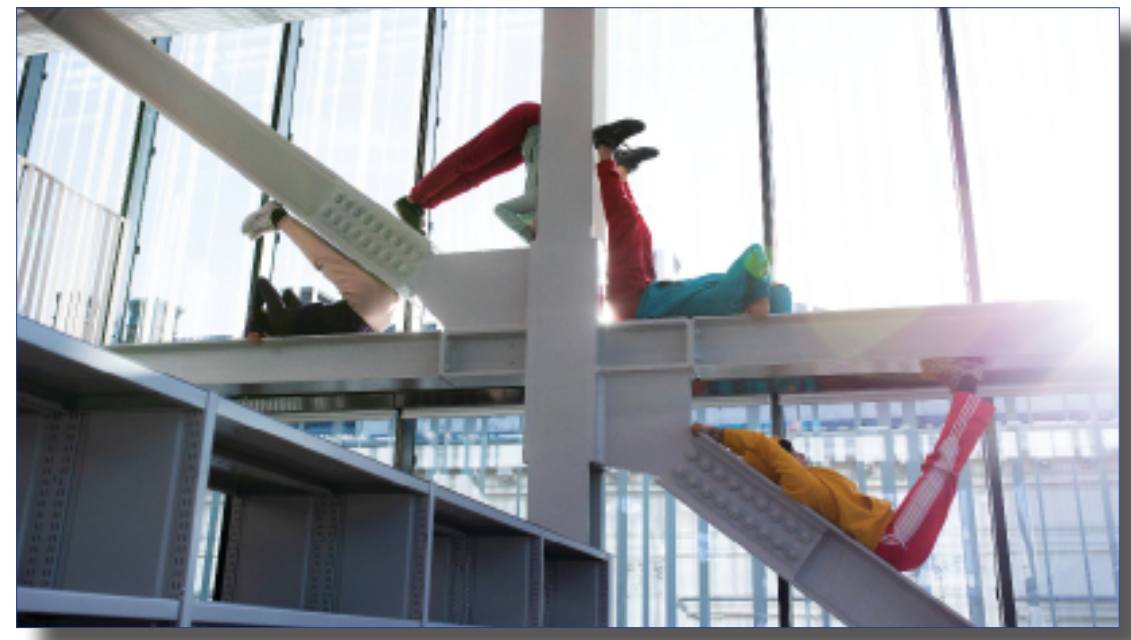

Abb. 6: SchauspielerInnen statt Bücher bei der Eröffnung; Foto: Universität Graz, Kanizaj)

Die besondere Eigenart des vorgegebenen Zeitplanes bestand darin, dass die Bibliothek unmittelbar nach der Eröffnung für zehn Tage wieder geschlossen werden musste, da zu diesem Zeitpunkt weder Sicherheit der Besucherlnnen, noch des Personals und auch nicht der Bestände gewährleistet werden konnte. In dieser kurzen Frist konnte natürlich nur ein geringer Teil der über 2.000 bis dahin festgestellten Baumängel behoben werden, dennoch bestand die Universitätsleitung auf die Inbetriebnahme des Hauses mit 7. Oktober.

\section{Aller Anfang ist schwer}

Der von außen betrachtet zumindest weitgehend intakte Zustand des Bauwerkes erweckte bei vielen Besucherlnnen in den ersten Tagen den völlig falschen Eindruck, dass es sich nicht nur um eine ästhetisch ansprechendes, sondern auch technisch und funktional ausgereiftes Objekt handle. In Wirklichkeit waren praktisch sämtliche Aufgaben, die eine Bibliothek im Regelbetrieb zu erfüllen hat, auch lange Zeit nach der Inbetriebnahme nur 
mit erheblichen Einschränkungen oder gar nicht wahrnehmbar. Große Teile des Gebäudes, wie die neuen Magazine, die Räume der Abteilung für Sondersammlungen inklusive Restaurierung und Digitalisierung, die Buchbinderwerkstätte sowie der 24/7-Bereich und die neuen Gruppenräume standen erst nach Monaten zur Verfügung, und der historische Lesesaal musste wiederholt für das Publikum gesperrt werden, weil die Arbeiten am Glasdach noch im Gange waren. An mehreren Stellen drang Wasser in die Räumlichkeiten, wodurch sich die Farbe von der Wand löste und der frisch verlegte Parkettboden aufwölbte. Die Klimasteuerung funktionierte unzureichend, am gesamten Gebäude fehlten Sonnenschutzeinrichtungen, die Garderobe konnte nicht in Betrieb genommen werden, die Buchförderanlage lief nur phasenweise und in einigen Bereichen - vor allem in den Magazinen - konnte das Licht nicht ausgeschaltet werden. Es existierte kein Leitsystem und die Lifte verzeichneten immer wieder Ausfälle oder waren längere Zeit durch Anlieferungen blockiert. Es gab keine intakte Videoüberwachung und es erforderte mehrere Anläufe, bis die Brandmeldeanlage abgenommen werden konnte.

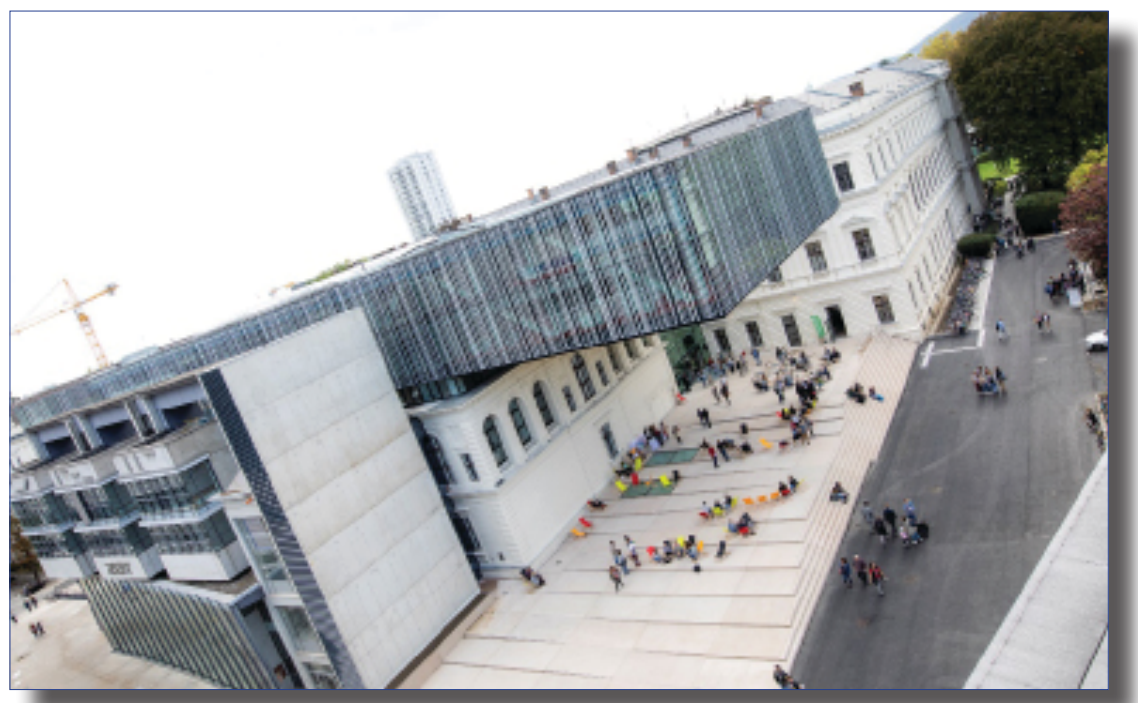

Abb. 7: Gesamtansicht des Gebäudes am Tag der Eröffnung (Foto: Universität Graz, Kanizaj)

Unter diesen Umständen ist es leicht vorstellbar, dass die Serviceleistungen der Bibliothek in den ersten Wochen und Monaten nach der Betriebsaufnahme nur mit großen Einschränkungen und mit großen Anstrengungen des Personals angeboten werden konnten. Erschwerend zu allen Unzuläng- 
lichkeiten kam hinzu, dass die zahlreichen im Haus tätigen ProfessionistInnen von unterschiedlichsten Firmen trotz wiederholter Unterweisung die Eröffnung der Bibliothek lange Zeit ignorierten. Immer wieder aufgekeilte Außentüren machten das Gebäude zu einem „open house“, Studierendenplätze - vor allem in den Gruppenräumen - wurden oft für Wochen mit Plänen und Werkzeug belegt, Lärm- und Geruchsbelästigungen standen auf der Tagesordnung.

Erstaunlicherweise wurden jedoch die hier nur beispielhaft aufgezählten Mängel von den Studierenden kaum wahrgenommen - im Gegenteil, die Räumlichkeiten wurden von Anfang an akzeptiert und in Beschlag genommen. Das vielfältige Angebot an unterschiedlichen Lern- und Arbeitssituationen fand vom ersten Augenblick an großen Anklang und es war überaus erfreulich zu sehen, dass die neue Universitätsbibliothek so großes Interesse erweckte.

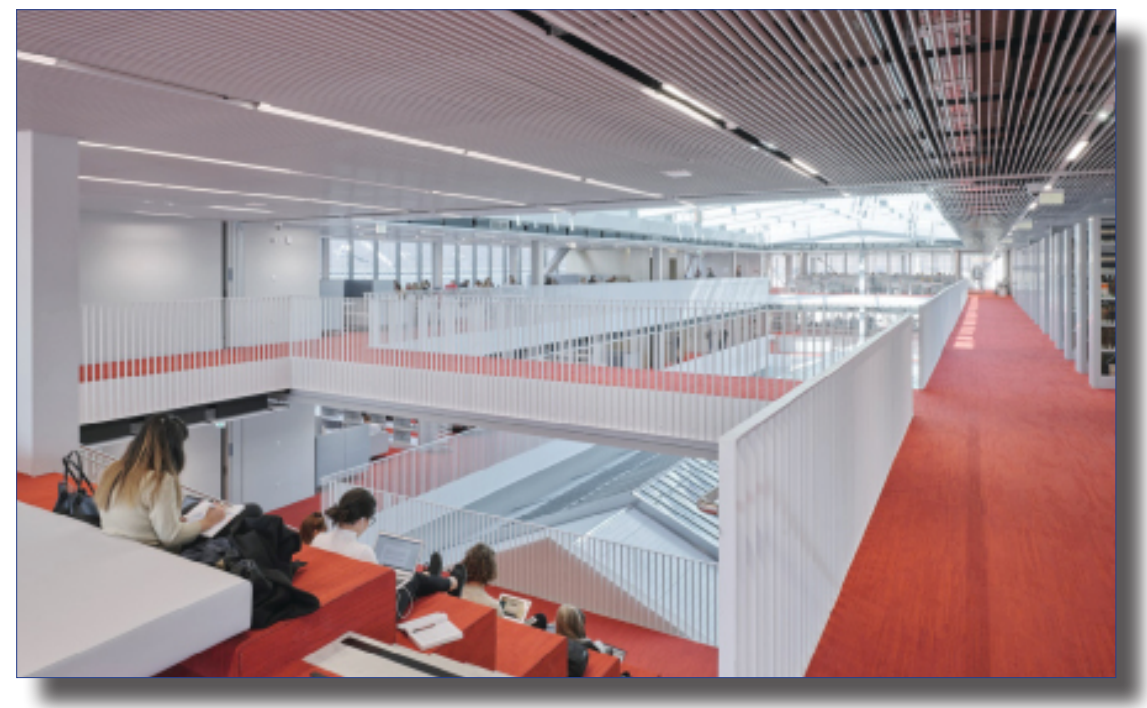

Abb. 8: Publikumsbereich 3. und 4. OG mit Sitzstiege im Vordergrund (Foto: Universität Graz, Schreyer)

\section{Epilog und Resümee}

Der vorliegende Beitrag entstand größtenteils im Februar 2020 und da ich von meinem Bürofenster direkt auf den Parkplatz mit den Firmenwagen blicken kann, kann ich behaupten, dass seit der Eröffnung im September 
vergangenen Jahres kein Tag vergangen ist, an dem nicht zumindest ein Trupp von Arbeiterlnnen im Gebäude tätig war, zumeist waren es mehrere. Auch wenn die Anzahl der Mängel täglich kleiner wird und die Anstrengungen der Beteiligten durchaus zu würdigen sind, kann die Situation nach wie vor nicht als zufriedenstellend angesehen werden. In den Energieferien wurden rund 2.300 Laufmeter Bücher aus unserem Außendepot in die Magazine auf dem Campus integriert, sodass die oft täglich mehrfach durchgeführten Abholfahrten reduziert werden konnten. Derzeit erfolgt auch die Planung für die Rückholung der Abteilung für Sondersammlungen mit ihren sehr umfangreichen Beständen. Dazu müssen die dafür vorgesehenen Regale neuerlich gereinigt und die Sicherheitseinrichtungen freigeschalten werden. In den letzten Tagen wurden auch die Digitalisierungs-, die Restaurierungs- und die Buchbindewerkstätten für den Bezug fertiggestellt. Die Buchbinderei wurde bereits in Betrieb genommen, für die beiden anderen Bereiche ist die Betriebsaufnahme für die Zeit nach Ostern vorgesehen. Vor Ende Mai müssen dann noch die sogenannten Rara-3-Bestände - ca. 5.000 Laufmeter - wieder rücktransportiert werden, die sie seit nunmehr fast fünfJahren in Schachteln verpackt und daher unbenützbar in verschiedenen Hallen untergebracht waren. Das Einstellen der Bände in die Regale wird aber frühestens in den Sommermonaten während der Ferien erfolgen können, da während des laufenden Semesterbetriebs die dafür notwendigen Personalkapazitäten nicht zur Verfügung stehen.

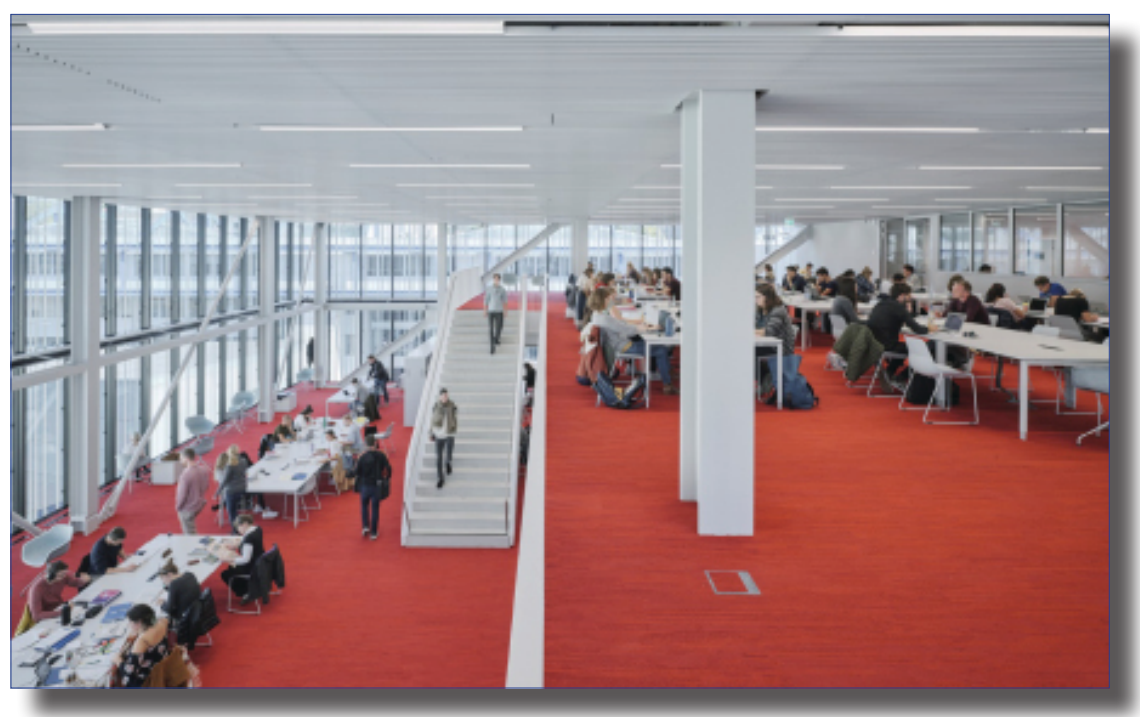

Abb. 9: Publikumsbereich 3. und 4. OG (Foto: Universität Graz, Schreyer) 
Für eine abschließende Beurteilung des Neu- und Umbauprojekts Universitätsbibliothek Graz ist es sicherlich noch zu früh, so liegt beispielweise noch keine finale Kostenabrechnung vor, die aber meiner Einschätzung nach noch zu einigen Diskussionen führen könnte, da mit beträchtlichen Überschreitungen des vorgegebenen Rahmens zu rechnen ist. Die laut der Rückmeldungen nahezu uneingeschränkte Akzeptanz des neuen Gebäudes durch die Studierenden sollte aber schon jetzt nicht dazu führen, die grundlegenden Fehler bei der Planung und Errichtung zu übersehen. Der vom Bauherrn, der Bundesimmobiliengesellschaft, von Beginn an ausgeübte Zeitdruck führte zu gravierenden Planungsmängeln, wodurch der ohnehin enge Zeitplan völlig aus den Fugen geriet. Das Ergebnis war nach meiner Beurteilung ein bei der Übergabe an die Bibliothek zu etwa siebzig Prozent fertiggestellter Bau, dessen volle Funktionalität etwa acht bis neun Monate nach der Eröffnung gegeben sein dürfte. Jetzt bleibt nur zu hoffen, dass das Gebäude auch bei zukünftigen Generationen von Studierenden als Lern- und Arbeitsstätte so beliebt bleibt und die unerfreulichen Umstände bei seiner Errichtung rasch in Vergessenheit geraten.

Dr. Werner Schlacher Universität Graz, Universitätsbibliothek E-Mail: werner.schlacher@uni-graz.at

* Den ersten und zweiten Teil des Berichtes finden Sie an folgenden Stellen: - Schlacher, W. (2016). Der Umbau der Universitätsbibliothek Graz ein Bericht in drei Fortsetzungen. Teil 1: Wie alles begann. Mitteilungen der Vereinigung Österreichischer Bibliothekarinnen und Bibliothekare 69(3-4), 422-435. https://doi.org/10.31263/voebm.v69i3.1732 - Schlacher, W. (2018). Der Umbau der Universitätsbibliothek Graz ein Bericht in drei Fortsetzungen - Teil 2: Vom Fall und Aufstieg eines Gebäudes". Mitteilungen der Vereinigung Österreichischer Bibliothekarinnen und Bibliothekare 71(3-4), 416-424. https://doi.org/10.31263/ voebm.v71i3-4.2162 